\title{
An exact expression of positive periodic solution for a first-order singular equation
}

Yun Xin ${ }^{1}$, Xiaoxiao Cui ${ }^{2}$ and Jie Liü ${ }^{2 *}$

${ }^{*}$ Correspondence:

jzyzliujie@hpu.edu.cn;

jeiliu_1985@126.com

${ }^{2}$ School of Mathematics and

Information Science, Henan

Polytechnic University, Jiaozuo

454000, China

Full list of author information is

available at the end of the article

\begin{abstract}
The main purpose of this paper is to obtain an exact expression of the positive periodic solution for a first-order differential equation with attractive and repulsive singularities. Moreover, we prove the existence of at least one positive periodic solution for this equation with an indefinite singularity by applications of topological degree theorem, and give the upper and lower bounds of the positive periodic solution.
\end{abstract}

MSC: $34 \mathrm{~B} 16 ; 34 \mathrm{~B} 18 ; 34 \mathrm{C} 25$

Keywords: First-order singular differential equation; Attractive and repulsive singularities; Indefinite singularity; Positive periodic solution

\section{Introduction}

A variety of population dynamics and physiological processes can be described by the following singular differential equation:

$$
x^{\prime}(t)+a(t) x(t)=\frac{b(t)}{x^{\rho}(t)}
$$

with periodic boundary value condition

$$
x(0)=x(\omega),
$$

where $a(t)$ and $b(t) \in C(\mathbb{R}, \mathbb{R})$ are $\omega$-periodic functions, $\rho$ and $\omega$ are positive constants. According to the literature [1-3], we say that Eq. (1.1) has an attractive singularity if $b(t)<0$, repulsive singularity if $b(t)>0$ and indefinite singularity if $b(t)$ may change sign. Moreover, we say that Eq. (1.1) has a strong singularity if $\rho \geq 1$ and a weak singularity if $0<\rho<1$.

As is well known, singular equations have a wide range of applications in many fields, and the existence of positive $\omega$-periodic solutions to singular equations plays a significant role in solving many practical problems. There is a good amount of work on periodic solutions for singular equations (see [4-18] and the references cited therein). In 2003, Agarwal and O'Regan [4] provided some results on positive $\omega$-periodic solutions of Eq. (1.1), where $b(t)>0$ (i.e., repulsive singularity). After that, Chu and Nieto [10] in 2008 proved the existence of positive $\omega$-periodic solutions for equation (1.1) with repulsive singularity and

(c) The Author(s) 2020. This article is licensed under a Creative Commons Attribution 4.0 International License, which permits use, sharing, adaptation, distribution and reproduction in any medium or format, as long as you give appropriate credit to the original author(s) and the source, provide a link to the Creative Commons licence, and indicate if changes were made. The images or other third party material in this article are included in the article's Creative Commons licence, unless indicated otherwise in a credit line to the material. If material is not included in the article's Creative Commons licence and your intended use is not permitted by statutory regulation or exceeds the permitted use, you will need to obtain permission directly from the copyright holder. To view a copy of this licence, visit http://creativecommons.org/licenses/by/4.0/. 
impulses by applications of Leray-Schauder alternative principle. Recently, Wang [15] and Chen et al. [7] discussed the existence of positive $\omega$-periodic solutions for the following singular differential equation:

$$
x^{\prime}(t)+a_{1}(t) x(t)=\lambda b_{1}(t) f(x(t))
$$

where $a_{1}(t)$ and $b_{1}(t) \in C(\mathbb{R},[0,+\infty))$ are $\omega$-periodic functions with $\int_{0}^{\omega} a_{1}(t) d t>0$ and $\int_{0}^{\omega} b_{1}(t) d t>0$, the nonlinear term $f$ has an attractive singularity or repulsive singularity. Their proofs were based on Krasnoselskii's fixed point theorem in a cone, the LeraySchauder degree and the upper and lower solutions method.

It is worth pointing out that the above results are only related to the existence of positive $\omega$-periodic solutions of first-order differential equations with attractive singularity or repulsive singularity, but the exact expression of $\omega$-periodic solutions is not involved.

First, in this paper we give an exact expression of positive $\omega$-periodic solution for Eq. (1.1) with attractive and repulsive singularities.

Theorem 1.1 Assume that condition $\int_{0}^{\omega} a(t) d t \cdot b(t)>0$ holds. Then Eq. (1.1) has a unique positive $\omega$-periodic solution $x(t)$, which can be written as

$$
x(t)=\left(\frac{(\rho+1) \int_{0}^{t} e^{(\rho+1) \int_{t}^{s} a(r) d r} b(s) d s}{1-e^{-(\rho+1) \int_{0}^{\omega} a(r) d r}}+\frac{(\rho+1) \int_{t}^{\omega} e^{(\rho+1) \int_{t}^{s} a(r) d r} b(s) d s}{e^{(\rho+1) \int_{0}^{\omega} a(r) d r}-1}\right)^{\frac{1}{\rho+1}} .
$$

Second, from the condition $\int_{0}^{\omega} a(t) d t \cdot b(t)>0$, it is easy to verify that the weight term $b(t)$ has a non-changing sign. A natural question is how Eq. (1.1) works on changing sign of $b(t)$ (i.e., indefinite singularity)? We also consider the existence of positive $\omega$-periodic solution for equation (1.1) with an indefinite singularity.

Theorem 1.2 Assume that $\bar{a}:=\frac{1}{\omega} \int_{0}^{\omega} a(t) d t>0$ and $\bar{b}:=\frac{1}{\omega} \int_{0}^{\omega} b(t) d t \geq 0$ hold. Furthermore, suppose the following conditions are satisfied:

$\left(H_{1}\right) 1-\frac{\|a\| \omega(\rho+1)}{2}>0$, here $\|a\|:=\max _{t \in[0, \omega]}|a(t)|$;

$\left(H_{2}\right) \frac{\bar{b}}{\overline{a_{+}}}-\frac{\omega(\rho+1)\left(\|a\| \overline{\left.\bar{t}_{+}+\|b\| \bar{a}\right)}\right.}{\bar{a}(2-\|a\| \omega(\rho+1))}>0$, here $a_{+}(t):=\max \{0, a(t)\}$.

Then Eq. (1.1) has at least one positive $\omega$-periodic solution $x(t)$ with

$$
x(t) \in\left[\left(\frac{\bar{b}}{\overline{a_{+}}}-\frac{\omega(\rho+1)\left(\|a\| \overline{b_{+}}+\|b\| \bar{a}\right)}{\bar{a}(2-\|a\| \omega(\rho+1))}\right)^{\frac{1}{\rho+1}},\left(\frac{\overline{b_{+}}}{\bar{a}}+\frac{\omega(\rho+1)\left(\|a\| \overline{b_{+}}+\|b\| \bar{a}\right)}{\bar{a}(2-\|a\| \omega(\rho+1))}\right)^{\frac{1}{\rho+1}}\right] .
$$

\section{Proof of Theorem 1.1}

In this section, an exact expression of solution for Eq. (1.1) with periodic boundary value condition is given.

First, we change the variable $x=u^{\alpha}$, where $\alpha=\frac{1}{\rho+1}$ and $0<\alpha<1$. Then Eq. (1.1) is converted into the following form:

$$
u^{\prime}(t)+\frac{a(t)}{\alpha} u(t)=\frac{b(t)}{\alpha},
$$

with periodic boundary value condition

$$
u(0)=u(\omega)
$$


Proof of Theorem 1.1 Applying method of variation of constant, solution of Eq. (2.1) can be written as the following form:

$$
u(t)=C(t) e^{-\int_{0}^{t} \frac{a(r)}{\alpha} d r},
$$

where $C(t) \in C(\mathbb{R}, \mathbb{R})$. Substituting Eq. (2.2) into Eq. (2.1), we arrive at

$$
C^{\prime}(t)=\frac{b(t)}{\alpha} e^{\int_{0}^{t} \frac{a(r)}{\alpha} d r} .
$$

Integrating the above equation over the interval $[0, t]$, here $t \in[0, \omega]$, we get

$$
C(t)=C(0)+\int_{0}^{t} \frac{b(s)}{\alpha} e^{\int_{0}^{s} \frac{a(r)}{\alpha} d r} d s
$$

Furthermore, we give

$$
C(\omega)=C(0)+\int_{0}^{\omega} \frac{b(s)}{\alpha} e^{\int_{0}^{s} \frac{a(r)}{\alpha} d r} d s
$$

Since $u(0)=u(\omega)$, we obtain

$$
C(0)=C(\omega) e^{-\int_{0}^{\omega} \frac{a(r)}{\alpha} d r}
$$

Combining Eqs. (2.4) and (2.5), we have

$$
C(0)=\frac{\int_{0}^{\omega} \frac{b(s)}{\alpha} e^{\int_{0}^{s} \frac{a(r)}{\alpha} d r} d s}{e^{\int_{0}^{\omega} \frac{a(r)}{\alpha} d r}-1}
$$

Then we get

$$
C(t)=\frac{\int_{0}^{\omega} \frac{b(s)}{\alpha} e^{\int_{0}^{s} \frac{a(r)}{\alpha} d r} d s}{e^{\int_{0}^{\omega} \frac{a(r)}{\alpha} d r}-1}+\int_{0}^{t} \frac{b(s)}{\alpha} e^{\int_{0}^{s} \frac{a(r)}{\alpha} d r} d s
$$

Therefore, the solution of Eq. (2.1) is

$$
\begin{aligned}
u(t) & =\left(\frac{\int_{0}^{\omega} \frac{b(s)}{\alpha} e^{\int_{0}^{s} \frac{a(r)}{\alpha} d r} d s}{e^{\int_{0}^{\omega} \frac{(a r)}{\alpha} d r}-1}+\int_{0}^{t} \frac{b(s)}{\alpha} e^{\int_{0}^{s} \frac{a(r)}{\alpha} d r} d s\right) e^{-\int_{0}^{t} \frac{a(r)}{\alpha} d r} \\
& =\int_{0}^{t} \frac{e^{\int_{t}^{s} \frac{a(r)}{\alpha} d r}}{1-e^{-\int_{0}^{\omega} \frac{a(r)}{\alpha} d r}} \frac{b(s)}{\alpha} d s+\int_{t}^{\omega} \frac{e^{\int_{t}^{s} \frac{a(r)}{\alpha} d r}}{e^{\int_{0}^{\omega} \frac{a(r)}{\alpha} d r}-1} \frac{b(s)}{\alpha} d s .
\end{aligned}
$$

Because $x(t)=u^{\alpha}(t)$ and $\alpha=\frac{1}{\rho+1}$, the solution of Eq. (1.1) is

$$
x(t)=\left(\frac{(\rho+1) \int_{0}^{t} e^{(\rho+1) \int_{t}^{s} a(r) d r} b(s) d s}{1-e^{-(\rho+1) \int_{0}^{\omega} a(r) d r}}+\frac{(\rho+1) \int_{t}^{\omega} e^{(\rho+1) \int_{t}^{s} a(r) d r} b(s) d s}{e^{(\rho+1) \int_{0}^{\omega} a(r) d r}-1}\right)^{\frac{1}{\rho+1}} .
$$

From the condition $\int_{0}^{\omega} a(t) d t \cdot b(t)>0$, it is easy to see that $x(t)$ is positive for all $t \in \mathbb{R}$. The proof is completed. 
Example 2.1 Consider the following first-order singular differential equation:

$$
x^{\prime}(t)+(\sin t+1) x(t)=\frac{e^{3 \cos t}}{x^{2}(t)}
$$

Comparing Eq. (2.7) with Eq. (1.1), we see that $a(t)=\sin t+1, b(t)=e^{3 \cos t}, \rho=2, \omega=2 \pi$. Furthermore, the condition

$$
\int_{0}^{\omega} a(t) d t \cdot b(t)=\int_{0}^{\pi}(\sin t+1) d t \cdot e^{3 \cos t}=2 \pi e^{3 \cos t}>0
$$

is satisfied. Using Eq. (2.6), we calculate the solution of Eq. (2.7) to be

$$
x(t)=e^{\cos t},
$$

and $x(t)$ is $2 \pi$-periodic. Substituting Eq. (2.8) into Eq. (2.7), we conclude that $e^{\cos t}$ is a solution of Eq. (2.7).

\section{Proof of Theorem 1.2}

For convenience, we recall the topological degree theorem by Mawhin [19].

Lemma 3.1 (see [19, Theorem 2.4]) Let $X, Z$ be real normed spaces and $L: D(L) \subset X \rightarrow Z$ a linear Fredholm map of index zero. Assume that $\Omega \subset X$ is an open bounded set and $N: \bar{\Omega} \rightarrow Z$ is an L-compact mapping. Furthermore, assume that the following conditions are satisfied:

(i) $L x+\lambda N x \neq 0$, for each $(x, \lambda) \in[(D(L) \backslash \operatorname{ker} L) \cap \partial \Omega] \times(0,1)$;

(ii) $N x \notin \operatorname{Im} L$, for each $x \in \operatorname{ker} L \cap \partial \Omega$;

(iii) $D_{0}\left(\left.Q N\right|_{\operatorname{ker} L}, \Omega \cap \operatorname{ker} L\right) \neq 0$, where $Q: Z \rightarrow Z$ is a continuous projector such that $\operatorname{ker} Q=\operatorname{Im} L$ and $D_{0}$ is the Brouwer degree.

Then the equation $L x+N x=0$ has at least one solution in $D(L) \cap \bar{\Omega}$.

Proof of Theorem 1.2 Define $X:=\{x \in C(\mathbb{R}, \mathbb{R}), x(t+\omega) \equiv x(t), \forall t \in \mathbb{R}\}$ endowed with the $C^{1}$-norm, $Z=L^{1}([0, \omega], \mathbb{R})$ with the $L^{1}$-norm. Let $D(L)=\left\{x \in X: x^{\prime}\right.$ is absolutely continuous on $\mathbb{R}$, we define operators $L: D(L) \rightarrow Z$ and $N: Z \rightarrow Z$ by

$$
(L x)(t)=x^{\prime}(t), \quad(N x)(t)=a(t) x(t)-\frac{b(t)}{x^{\rho}(t)} .
$$

Form this, Eq. (1.1) can be converted to $L x+N x=0$. Define projectors $P: X \rightarrow X$ and $Q: Z \rightarrow Z$ by

$$
P x=\frac{1}{\omega} \int_{0}^{\omega} x(s) d s, \quad Q z=\frac{1}{\omega} \int_{0}^{\omega} z(s) d s
$$

As literature [20, Theorem 1.1], it is easy to verify that $L$ is a Fredholm linear mapping with zero index and for any open bounded set $\Omega \subset X, N$ is $L$-compact on $\bar{\Omega}$. 
Consider the following homotopy family of Eq. (1.1):

$$
x^{\prime}(t)+\lambda a(t) x(t)=\lambda \frac{b(t)}{x^{\rho}(t)}, \quad \lambda \in(0,1],
$$

which can be expressed as the abstract equation $L x+\lambda N x=0$.

Multiplying both sides of Eq. (3.2) with $x^{\rho}(t)$ and integrating over the interval $[0, \omega]$, we get

$$
\int_{0}^{\omega} a(t) x^{\rho+1}(t) d t=\int_{0}^{\omega} b(t) d t=\bar{b} \omega,
$$

which yields

$$
\int_{0}^{\omega} a_{+}(t) x^{\rho+1}(t) d t \geq \bar{b} \omega .
$$

Thus, there exists a point $\eta \in[0, \omega]$ such that

$$
x^{\rho+1}(\eta) \overline{a_{+}} \omega \geq \bar{b} \omega .
$$

Since $\bar{b} \geq 0$, we obtain

$$
x(\eta) \geq\left(\frac{\bar{b}}{\overline{a_{+}}}\right)^{\frac{1}{\rho+1}} .
$$

Meanwhile, Eq. (3.2) together with the fact of $x(t)>0$ gives

$$
\frac{x^{\prime}(t)}{x(t)}+\lambda a(t)=\lambda \frac{b(t)}{x^{\rho+1}(t)} .
$$

Integrating the above equation over the interval $[0, \omega]$, we get

$$
\bar{a} \omega=\int_{0}^{\omega} a(t) d t=\int_{0}^{\omega} \frac{b(t)}{x^{\rho+1}(t)} d t,
$$

which yields

$$
\bar{a} \omega \leq \int_{0}^{\omega} \frac{b_{+}(t)}{x^{\rho+1}(t)} d t .
$$

Thus, there exists a point $\xi \in[0, \omega]$ such that

$$
\bar{a} \omega \leq \frac{\overline{b_{+}} \omega}{x^{\rho+1}(\xi)} .
$$

Since $\bar{a}>0$, we arrive at

$$
x(\xi) \leq\left(\frac{\overline{b_{+}}}{\bar{a}}\right)^{\frac{1}{\rho+1}} .
$$


Let $x=u^{\alpha}$, where $\alpha=\frac{1}{\rho+1}$, Eqs. (3.2), (3.3) and (3.4) can be turned into

$$
\begin{aligned}
& u^{\prime}(t)+\lambda \frac{a(t)}{\alpha} u(t)=\lambda \frac{b(t)}{\alpha}, \\
& u(\eta)=x^{\rho+1}(\eta) \geq \frac{\bar{b}}{\overline{a_{+}}} \\
& u(\xi)=x^{\rho+1}(\xi) \leq \frac{\overline{b_{+}}}{\bar{a}} .
\end{aligned}
$$

It follows from Eqs. (3.6) and (3.7) that

$$
\begin{aligned}
u(t) & =\frac{1}{2}(u(t)+u(t-\omega)) \\
& =\frac{1}{2}\left(u(\eta)+\int_{\eta}^{t} u^{\prime}(s) d s+u(\eta)-\int_{t-\omega}^{\eta} u^{\prime}(s) d s\right) \\
& \geq u(\eta)-\frac{1}{2}\left(\int_{t-\omega}^{\eta}\left|u^{\prime}(s)\right| d s+\int_{\eta}^{t}\left|u^{\prime}(s)\right| d s\right) \\
& \geq \frac{\bar{b}}{\overline{a_{+}}}-\frac{1}{2} \int_{0}^{\omega}\left|u^{\prime}(t)\right| d t .
\end{aligned}
$$

Similarly, we deduce

$$
\begin{aligned}
u(t) & =u(\xi)+\frac{1}{2}\left(\int_{\xi}^{t} u^{\prime}(s) d s-\int_{t-\omega}^{\xi} u^{\prime}(s) d s\right) \\
& \leq u(\xi)+\frac{1}{2}\left(\int_{\xi}^{t}\left|u^{\prime}(s)\right| d s+\int_{t-\omega}^{\xi}\left|u^{\prime}(s)\right| d s\right) \\
& \leq \frac{\overline{b_{+}}}{\bar{a}}+\frac{1}{2} \int_{0}^{\omega}\left|u^{\prime}(t)\right| d t .
\end{aligned}
$$

Multiplying both sides of Eq. (3.5) with $u^{\prime}(t)$ and integrating it over the interval $[0, \omega]$, we get

$$
\begin{aligned}
\int_{0}^{\omega}\left|u^{\prime}(t)\right|^{2} d t & =-\lambda \int_{0}^{\omega} \frac{a(t)}{\alpha} u(t) u^{\prime}(t) d t+\lambda \int_{0}^{\omega} \frac{b(t)}{\alpha} u^{\prime}(t) d t \\
& \leq \frac{\|a\|}{\alpha} \int_{0}^{\omega}|u(t)|\left|u^{\prime}(t)\right| d t+\frac{\|b\|}{\alpha} \int_{0}^{\omega}\left|u^{\prime}(t)\right| d t .
\end{aligned}
$$

From Eq. (3.9) and the Hölder inequality, the above equation implies

$$
\begin{aligned}
\int_{0}^{\omega}\left|u^{\prime}(t)\right|^{2} d t & \leq\left(\frac{\overline{b_{+}}}{\bar{a}}+\frac{1}{2} \int_{0}^{\omega}\left|u^{\prime}(t)\right| d t\right) \frac{\|a\|}{\alpha} \int_{0}^{\omega}\left|u^{\prime}(t)\right| d t+\frac{\|b\|}{\alpha} \int_{0}^{\omega}\left|u^{\prime}(t)\right| d t \\
& =\frac{\|a\|}{2 \alpha}\left(\int_{0}^{\omega}\left|u^{\prime}(t)\right| d t\right)^{2}+\left(\frac{\|a\| \overline{b_{+}}}{\alpha \bar{a}}+\frac{\|b\|}{\alpha}\right) \int_{0}^{\omega}\left|u^{\prime}(t)\right| d t \\
& \leq \frac{\|a\| \omega}{2 \alpha} \int_{0}^{\omega}\left|u^{\prime}(t)\right|^{2} d t+\left(\frac{\|a\| \overline{b_{+}}}{\alpha \bar{a}}+\frac{\|b\|}{\alpha}\right) \omega^{\frac{1}{2}}\left(\int_{0}^{\omega}\left|u^{\prime}(t)\right|^{2} d t\right)^{\frac{1}{2}} .
\end{aligned}
$$


It follows from condition $\left(H_{1}\right)$ and the above inequality that

$$
\left(\int_{0}^{\omega}\left|u^{\prime}(t)\right|^{2} d t\right)^{\frac{1}{2}} \leq \frac{\left(\frac{\|a\| \overline{b_{+}}}{\alpha \bar{a}}+\frac{\|b\|}{\alpha}\right) \omega^{\frac{1}{2}}}{1-\frac{\|a\| \omega}{2 \alpha}}=\frac{2 \omega^{\frac{1}{2}}\left(\|a\| \overline{b_{+}}+\|b\| \bar{a}\right)}{\bar{a}(2 \alpha-\|a\| \omega)} .
$$

Using Eqs. (3.8), (3.9) and (3.10), we obtain

$$
u(t) \geq \frac{\bar{b}}{\overline{a_{+}}}-\frac{\omega^{\frac{1}{2}}}{2}\left(\int_{0}^{\omega}\left|u^{\prime}(t)\right|^{2} d t\right)^{\frac{1}{2}} \geq \frac{\bar{b}}{\overline{a_{+}}}-\frac{\omega\left(\|a\| \overline{b_{+}}+\|b\| \bar{a}\right)}{\bar{a}(2 \alpha-\|a\| \omega)}>0
$$

since condition $\left(H_{2}\right)$. Moreover,

$$
u(t) \leq \frac{\overline{b_{+}}}{\bar{a}}+\frac{\omega^{\frac{1}{2}}}{2}\left(\int_{0}^{\omega}\left|u^{\prime}(t)\right|^{2} d t\right)^{\frac{1}{2}} \leq \frac{\overline{b_{+}}}{\bar{a}}+\frac{\omega\left(\|a\| \overline{b_{+}}+\|b\| \bar{a}\right)}{\bar{a}(2 \alpha-\|a\| \omega)} .
$$

Therefore, we obtain

$$
x(t)=u^{\frac{1}{\rho+1}}(t) \geq\left(\frac{\bar{b}}{\overline{a_{+}}}-\frac{\omega(\rho+1)\left(\|a\| \overline{b_{+}}+\|b\| \bar{a}\right)}{\bar{a}(2-\|a\| \omega(\rho+1))}\right)^{\frac{1}{\rho+1}}:=M_{1}>0
$$

and

$$
x(t)=u^{\frac{1}{\rho+1}}(t) \leq\left(\frac{\overline{b_{+}}}{\bar{a}}+\frac{\omega(\rho+1)\left(\|a\| \overline{b_{+}}+\|b\| \bar{a}\right)}{\bar{a}(2-\|a\| \omega(\rho+1))}\right)^{\frac{1}{\rho+1}}:=M_{2} .
$$

Define

$$
\Omega_{1}:=\left\{x \in X: E_{1}<x(t)<E_{2}, \forall t \in \mathbb{R}\right\}
$$

where $0<E_{1}<M_{1}, E_{2}>M_{2}$. Then conditions (i) and (ii) of Lemma 3.1 are verified. For a constant $x \in \operatorname{ker} L, x>0$, we have

$$
Q N x=\frac{1}{\omega} \int_{0}^{\omega}\left(a(t) x(t)-\frac{b(t)}{x^{\rho}(t)}\right) d t .
$$

It follows from Eqs. (3.3), (3.4), (3.11) and (3.12) that

$$
\int_{0}^{\omega}\left(a(t) E_{1}-\frac{b(t)}{E_{1}^{\rho}}\right) d t \cdot \int_{0}^{\omega}\left(a(t) E_{2}-\frac{b(t)}{E_{2}^{\rho}}\right) d t<0 .
$$

Therefore, we obtain

$$
D_{0}\left(\left.Q N\right|_{\text {ker } L}, \Omega_{1} \cap \operatorname{ker} L\right)=1 \text {. }
$$

Thus condition (iii) of Lemma 3.1 holds. Therefore, $L x+N x=0$ has at least one solution in $\bar{\Omega}_{1}$, which means Eq. (1.1) has at least one positive $\omega$-periodic solution $x(t)$ with

$$
x(t) \in\left[\left(\frac{\bar{b}}{\overline{a_{+}}}-\frac{\omega(\rho+1)\left(\|a\| \overline{b_{+}}+\|b\| \bar{a}\right)}{\bar{a}(2-\|a\| \omega(\rho+1))}\right)^{\frac{1}{\rho+1}},\left(\frac{\overline{b_{+}}}{\bar{a}}+\frac{\omega(\rho+1)\left(\|a\| \overline{b_{+}}+\|b\| \bar{a}\right)}{\bar{a}(2-\|a\| \omega(\rho+1))}\right)^{\frac{1}{\rho+1}}\right] .
$$


Example 3.1 Consider the following first-order differential equation with an indefinite singularity:

$$
x^{\prime}(t)+\left(\frac{1}{16} \cos (16 t)+\frac{1}{32}\right) x(t)=\frac{\frac{1}{32} \sin (16 t)+\frac{\sqrt{3}}{64}}{x^{3}(t)} .
$$

Comparing Eq. (3.13) with Eq. (1.1), we see that $a(t)=\frac{1}{16} \cos (16 t)+\frac{1}{32}, b(t)=\frac{1}{32} \sin (16 t)+$ $\frac{\sqrt{3}}{64}, \rho=3, \omega=\frac{\pi}{8}$. By calculating, we get

$$
\begin{aligned}
& \|a\|=\frac{3}{32}, \quad \bar{a}=\frac{1}{32}, \quad \overline{a_{+}}=\frac{6 \sqrt{3}+\pi}{96 \pi}, \\
& \|b\|=\frac{2+\sqrt{3}}{64}, \quad \bar{b}=\frac{\sqrt{3}}{64}, \quad \overline{b_{+}}=\frac{3+\sqrt{3} \pi}{96 \pi} .
\end{aligned}
$$

It can be verified that conditions $\left(H_{1}\right)$ and $\left(H_{2}\right)$ are satisfied. Using Theorem 1.2, we see that Eq. (3.13) has at least one positive $\frac{\pi}{8}$-periodic solution $x(t)$ with

$$
x(t) \in\left[\left(\frac{3 \sqrt{3} \pi}{2(6 \sqrt{3}+\pi)}-\frac{6+2 \pi+3 \sqrt{3} \pi}{2(128-3 \pi)}\right)^{\frac{1}{4}},\left(\frac{3+\sqrt{3} \pi}{3 \pi}+\frac{6+2 \pi+3 \sqrt{3} \pi}{2(128-3 \pi)}\right)^{\frac{1}{4}}\right] .
$$

\section{Conclusions}

In this paper, applying method of variation of constant, we obtain an exact expression of positive periodic solution for equation (1.1) with attractive and repulsive type. After that, we prove the existence of at least one positive periodic solution for Eq. (1.1) with an indefinite singularity by applications of topological degree theorem, and give the upper and lower bound of the positive periodic solution.

\section{Acknowledgements}

$Y X, X X C, J L$ are grateful to anonymous referees for their constructive comments and suggestions which have greatly improved this paper.

\section{Funding}

This work was supported by Research is supported by Key Research Funds for the Universities of Henan Province (19A110018, 20B110006), Fundamental Research Funds for the Universities of Henan Province (NSFRF170302, NSFRF180320), Henan Polytechnic University Doctor Fund (B2016-58).

\section{Abbreviations}

Not applicable.

Availability of data and materials

Not applicable.

\section{Ethics approval and consent to participate}

$Y X, X X C, J L$ contributed to each part of this study equally and declare that they have no competing interests.

\section{Competing interests}

$Y X, X X C$, JL declare that they have no competing interests.

\section{Consent for publication}

$Y X, X X C$, JL read and approved the final version of the manuscript.

\section{Authors' contributions}

$Y X, X X C$, JL contributed equally and significantly in writing this article. All authors read and approved the final manuscript.

\section{Author details}

${ }^{1}$ College of Computer Science and Technology, Henan Polytechnic University, Jiaozuo 454000, China. ${ }^{2}$ School of Mathematics and Information Science, Henan Polytechnic University, Jiaozuo 454000, China. 


\section{Publisher's Note}

Springer Nature remains neutral with regard to jurisdictional claims in published maps and institutional affiliations.

Received: 23 July 2020 Accepted: 16 September 2020 Published online: 29 September 2020

\section{References}

1. Lazer, A., Solimini, S.: On periodic solutions of nonlinear differential equations with singularities. Proc. Am. Math. Soc. 99, 109-114 (1987)

2. Torres, P.: Mathematical Models with Singularities-A Zoo of Singular Creatures. Atlantis Briefs in Differential Equations. Atlantis Press, Paris (2015)

3. Cheng, Z., Ren, J: Periodic solution for second order damped differential equations with attractive-repulsive singularities. Rocky Mt. J. Math. 48, 753-768 (2018)

4. Agarwal, A., O'Regan, D.: Singular Differential and Integral Equations with Applications. Kluwer Academic, Dordrecht (2003)

5. Bartsch, T., Gebhard, B.: Global continua of periodic solutions of singular first-order Hamiltonian systems of $\mathrm{N}$-vortex type. Math. Ann. 369, 627-651 (2017)

6. Bai, D., Xu, Y.: Periodic solutions of first order functional differential equations with periodic deviations. Comput. Math. Appl. 53, 1361-1366 (2007)

7. Chen, R., Ma, R., He, Z.: Positive periodic solutions of first-order singular systems. Appl. Math. Comput. 218, $11421-11428(2012)$

8. Cheng, Z., Yuan, Q.: Damped superlinear Duffing equation with strong singularity of repulsive type. J. Fixed Point Theory Appl. 22, 37 (2020)

9. Cheng, Z., Li, F.: Positive periodic solutions for a kind of second-order neutral differential equations with variable coefficient and delay. Mediterr. J. Math. 15, 134 (2018)

10. Chu, J., Nieto, J.: Impulsive periodic solutions of first-order singular differential equations. Bull. Lond. Math. Soc. 40 , 143-150 (2008)

11. Kong, F., Luo, Z.: Positive periodic solutions for a kind of first-order singular differential equation induced by impulses. Qual. Theory Dyn. Syst. 17, 375-386 (2018)

12. LV, L., Cheng, Z:: Positive periodic solution to superlinear neutral differential equation with time-dependent parameter. Appl. Math. Lett. 98, 271-277 (2019)

13. Nieto, J., Uzal, J.: Pulse positive periodic solutions for some classes of singular nonlinearities. Appl. Math. Lett. 86 134-140 (2018)

14. Sun, X., Yu, P.: Periodic traveling waves in a generalized BBM equation with weak backward diffusion and dissipation terms. Discrete Contin. Dyn. Syst., Ser. B 24, 965-987 (2019)

15. Wang, H.: Positive periodic solutions of singular systems of first order ordinary differential equations. Appl. Math. Comput. 218, 1605-1610 (2011)

16. Xin, Y., Liu, H.: Singularities of attractive and repulsive type for $p$-Laplacian generalized Liénard equation. Adv. Differ. Equ. 2018, 471 (2018)

17. Xin, Y., Hu, G.: Periodic solution for prescribed mean curvature Rayleigh equation with a singularity. Adv. Differ. Equ. $2020,269(2020)$

18. Zhang, X., Li, X., Jiang, D., Wang, K.: Multiplicity positive solutions to periodic problems for first-order impulsive differential equations. Comput. Math. Appl. 52, 953-966 (2006)

19. Mawhin, J: Topological degree and boundary value problems for nonlinear differential equations. In: Topological Methods for Ordinary Differential Equations (Montecatini Terme, 1991). Lecture Notes in Math., vol. 1537, pp. 74-142. Springer, Berlin (1993)

20. Xin, Y., Cheng, Z: Positive periodic solution to indefinite singular Liénard equation. Positivity 23, 779-787 (2019)

\section{Submit your manuscript to a SpringerOpen ${ }^{\circ}$ journal and benefit from:}

- Convenient online submission

- Rigorous peer review

- Open access: articles freely available online

- High visibility within the field

- Retaining the copyright to your article

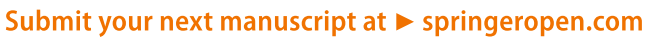

